\title{
ANÁLISE DE DADOS HISTÓRICOS DE RADIAÇÃO SOLAR ACUMULADA COLETADOS PELA PCD DE ILHÉUS-BA
}

\author{
Ykaro da Cruz Pereira ${ }^{(a)}$, Ronaldo Lima Gomes ${ }^{(b)}$, Adriano Hoth Cerqueira ${ }^{(c)}$ \\ (a) Departamento de Ciências Agrárias e Ambientais/ Universidade Estadual de Santa Cruz, ykarodacruz@ gmail.com \\ (b) Departamento de Ciências Agrárias e Ambientais/Universidade Estadual de Santa Cruz, rlgomes.uesc@ gmail.com \\ (c) Departamento de Ciências Exatas e Tecnológicas/ Universidade Estadual de Santa Cruz, hoth@uesc.br
}

Eixo: Climatologia em diferentes níveis escalares: mudanças e variabilidades

\begin{abstract}
Resumo
Este projeto possui o objetivo de analisar dados históricos de radiação solar acumulada, coletados pela Plataforma de Coleta de Dados Meteorológicos - PCD, localizada no Município de Ilhéus-BA, objetivando subsidiar as atividades de execução do projeto Mapeamento do potencial solar para microgeração de energia elétrica em ambiente urbano: o caso da cidade de Ilhéus-Ba. O método adotado iniciou-se com o acesso ao banco de dados do SINDA - Sistema Integrado de dados Ambientais do INPE - Instituto Nacional de Pesquisas Espaciais, na área de dados históricos coletados por PCDs espalhadas pelo território brasileiro. Em seguida os dados obtidos em formato de planilha foram filtrados e analisados, correspondentes ao período de 18/10/1997 até 15/10/2016, tendo em vista o entendimento da incidência de radiação solar acumulada no período estudado. Tais dados serão úteis para compor o panorama do potencial solar para geração de energia elétrica na área de abrangência da cidade de Ilhéus-BA.
\end{abstract}

Palavras chave: Radiação Solar, Coleta de dados, Energia fotovoltaica, Ilhéus.

\section{Introdução}

O presente artigo trata-se de um projeto de pesquisa de iniciação científica, em andamento, subsidiado pela Fundação de Amparo à Pesquisa do Estado da Bahia - FAPESB, que tem como objetivo analisar e tratar dados históricos de radiação solar acumulada disponíveis na Plataforma de Coleta de Dados Meteorológicos - PCD, n 32499, localizada no Centro de Pesquisas do Cacau - CEPEC, Ilhéus-BA, nas

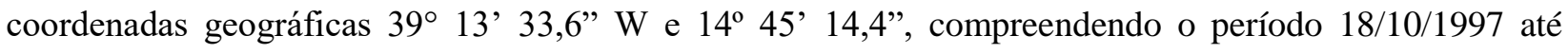
15/10/2016, visando compor o macroprojeto: Mapeamento do potencial solar para microgeração de energia elétrica em ambiente urbano: o caso da cidade de Ilhéus-BA.

As PCDs são estações utilizadas para obter regularmente informações colhidas em lugares remotos ou espalhadas por uma região muito extensa, podendo-se lograr informações meteorológicas e são utilizadas para previsão do tempo e estudos climáticos (INPE, 2017; INEMA, 2011). 
Em particular, neste trabalho, apresentamos a variação anual do valor médio mensal dos valores compilados da irradiação solar global. Em uma primeira abordagem, comparamos estes valores com o resultado de um modelo numérico que leva em consideração a variação dos parâmetros orbitais terrestres ao longo do ano e um modelo simplificado de transmitância para a atmosfera terrestre. Os resultados demostram que este modelo reproduz de forma satisfatória a forma da curva medida em solo, e discute-se potenciais melhorias do modelo, visando a compreensão da estrutura fina dos dados observados e suas implicações para a estimativa do potencial de geração de energia local.

\subsection{Revisão de Literatura}

No Brasil, a matriz energética é predominantemente renovável. A utilização de fontes não renováveis para geração de energia proporciona mais poluição e, portanto, maiores são os impactos ambientais (HERMANN e CAMARA, 2016; HONDA, 2015, p. 1). Nesse contexto de procura de modelos sustentáveis que diminuam os impactos ambientais e proporcionem a preservação dos tipos de vida na Terra, a energia solar fotovoltaica se destaca, como sendo a principal forma de geração de energia limpa, adicionando-se o fato de que o Brasil "recebe uma intensa radiação solar durante o ano, com destaque para região nordeste, que possui o maior índice de radiação solar" (HONDA, 2015). Porém, as usinas solares não chegam a 0,5 da matriz renovável, onde no Brasil, a matriz energética distribui-se em 70,6\% Hidráulica; 11,3\% - Gás Natural; 7,6\% - Biomassa; 4,4\% - Derivados de Petróleo; 2,6\% - Carvão e Derivados; 2,4\% - Nuclear e 1,1\% - Eólica (EPE, 2014).

A partir da regularização governamental dada pela Resolução ANEEL 482/2012, a micro geração de energia solar vem se disseminado cada vez mais, em virtude do declínio dos custos de instalação e aumento dos incentivos fiscais. A Resolução 482/2012 permite o uso de "micro e mini geração distribuída de energia fotovoltaica com sistema de compensação e conectadas a rede elétrica pública" (ANEEL, 2012). Assim, é permitido que a energia excedente, gerada pela unidade consumidora, seja injetada na rede de distribuidora, contabilizando um crédito em energia (KWh) a ser abatido na fatura (HONDA, 2015). De acordo com Ordones (2017), a energia solar vem se tornando fonte complementar de geração à energia hidrelétrica e a estimativa, com base em projeções feitas pela Empresa de Pesquisa Energética EPE, a estimativa é que a sua participação na matriz energética brasileira será de mais de $30 \%$ em 2030 , onde 8,2 dos 25 giga watts $(\mathrm{GW})$ se darão por geração distribuída em casas, edifícios comerciais e públicos, condomínios e na área rural. Wanderley e Campos (2013, p. 11), analisam que "a produção residencial de energia solar já é viável para 15\% dos domicílios brasileiros, cujo custo de geração de um equipamento de pequena potência é mais barato que a energia vendida por 10 das mais de 60 distribuidoras do país". 


\section{Metodologia}

A irradiação solar global $\left(\mathrm{em} \mathrm{MJ} / \mathrm{m}^{2}\right)$ que atinge a superfície da Terra é dada pela soma de componentes: direta e difusa. A componente direta é obtida através da integração da radiação oriunda apenas do ângulo sólido compreendido pelo disco solar. Já a radiação difusa é obtida através da integração (em ângulo sólido) de todo o semi-hemisfério (isto é, $2 \pi$ ), descontando-se, evidentemente, o disco solar. Esta diferenciação (na definição e no processo de medida) é importante porque nem todas as PCDs medem a radiação direta. A estação em Ilhéus, por exemplo, fornece apenas a componente global, e é, portanto, desta componente que trataremos exclusivamente aqui.

As estações PCDs disponibilizam para cada intervalo de 3 horas, numa integração de 1080 amostras a cada 10 segundos de intervalo, a intensidade de energia que chega à superfície da Terra ou irradiação solar global incidente (em uma superfície plana; em $\mathrm{MJ} / \mathrm{m}^{2}$ ). Para cada dia do ano, somamos todas as medidas e convertemos o valor resultante para $\mathrm{kWh} / \mathrm{m}^{2}$ (como o objetivo final deste trabalho é avaliar o potencial de geração de energia a partir de placas fotovoltaica no município de Ilhéus, esta é a unidade física relevante para convertemos a área coberta pelos módulos/placas em quantidade de energia a ser injetada no sistema). Para cada mês do ano obteve-se um valor médio (ver Figura 2). Foi realizado, também um modelo numérico baseado em variáveis puramente astronômicas, para obtermos uma curva teórica de irradiação solar extraterrestre, isto é, sem interferências da atmosfera. Para isto, assumiu-se um valor para a constante solar de $S_{0}=1362 \mathrm{~W} / \mathrm{m}^{2}$. Para cada dia do ano, foi calculado o valor da distância Terra-Sol e calculou-se, também, um valor para a irradiação solar extraterrestre, $\mathrm{S}=S_{0}(1 / r)^{2}$, na posição da Terra (nesta equação, $r$ é dado em unidades astronômicas). Para estimar o valor que chega na superfície (da irradiação global), foram adotadas duas hipóteses: 1) o valor da irradiação que chega na superfície da Terra, $S$, pode ser parametrizado a partir da definição de transparência atmosférica: $S_{\text {solo }} / S_{0}=k, 2$ ) e que podemos estimar um valor médio anual para $k$ utilizando-se dos dados disponíveis no SWERA.

\section{Resultados}

Com a organização e tratamento dos dados, observaram-se as datas e horários de coleta, sendo que em alguns períodos não havia constância de informações. Tais informações foram subsídios para a formulação de gráficos e tabelas indicadores do índice de radiação numa média correspondente aos valores acumulados diário, mensal e anual. Elaboraram-se gráficos a partir dos dados que datam aos períodos que correspondem aos anos de 2000-2003, 2005-2012 e 2013-2016, observando-se que nesses anos não houve interrupções consideráveis na disponibilização de informações da PCD. 


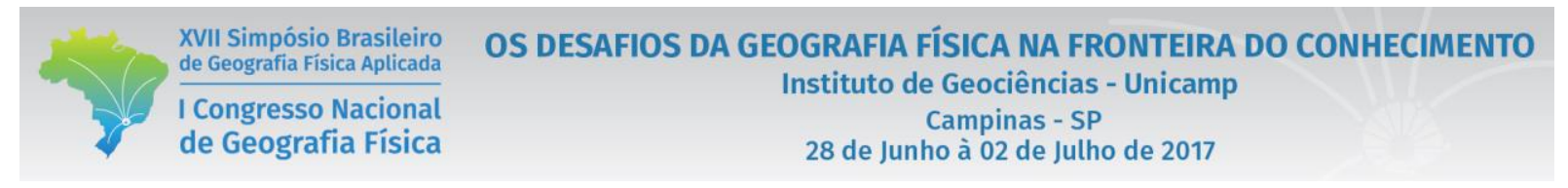

O mapa do valor médio anual para a irradiação global que chega ao município de Ilhéus pode ser visto na Figura 1. Adotamos o valor de $4.98 \mathrm{kWh} / \mathrm{m}^{2}$ como valor médio. Paralelamente, ajustamos o valor da transparência atmosférica $\mathrm{k}$ de modo que a curva diária para a irradiação resultasse em um valor médio idêntico aos fornecidos pelos mapas do SWERA. Obtivemos, então, $k=0.512$. Com este valor, calculamos numericamente o valor da irradiação esperada em solo e a comparamos com os dados do PCD.

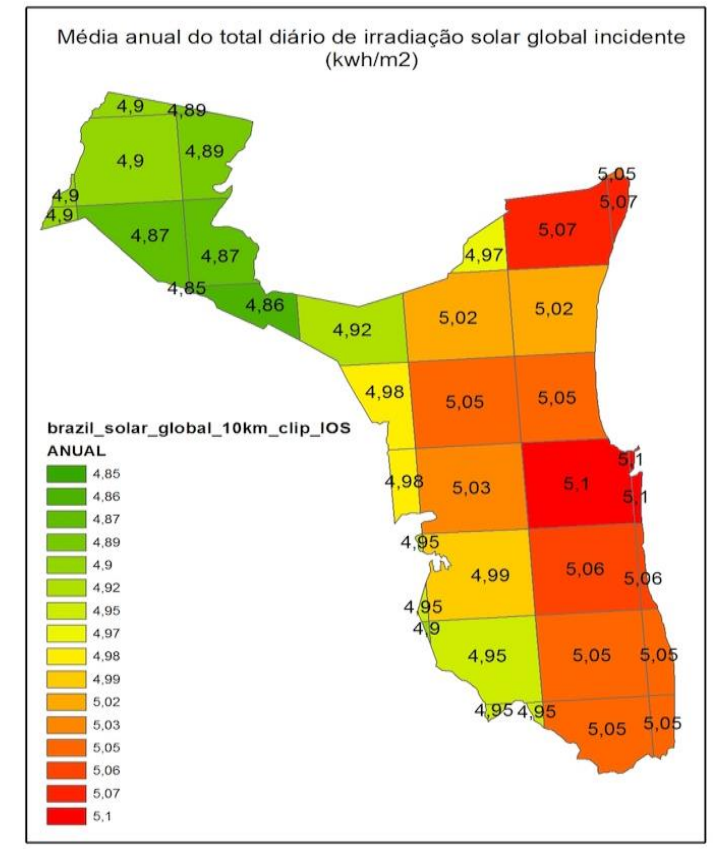

Figura 1 - Mapa do valor médio anual para a irradiação global em Ilhéus. Fonte: Atlas Brasileiro de Energia Solar,2006.

Na figura 2, mostramos a curva teórica (curva sólida) e a curva dos dados do PCD (curva vermelha). Nota-se que será preciso ajustes mais finos das hipóteses de trabalho (associação de um valor médio para k a partir dos dados obtidos pelo SWERA), além da análise estatística dos dados da estação, uma vez que apenas o ano de 2015 está descrito neste gráfico. Ressalta-se contudo, que discrepância entre as curvas se dá em intensidade, e é razoável supor que um melhor ajuste do parâmetro $k$ deva ser capaz de reproduzir bem a curva observada. Esta etapa do trabalho será apresentada a posteriori. 


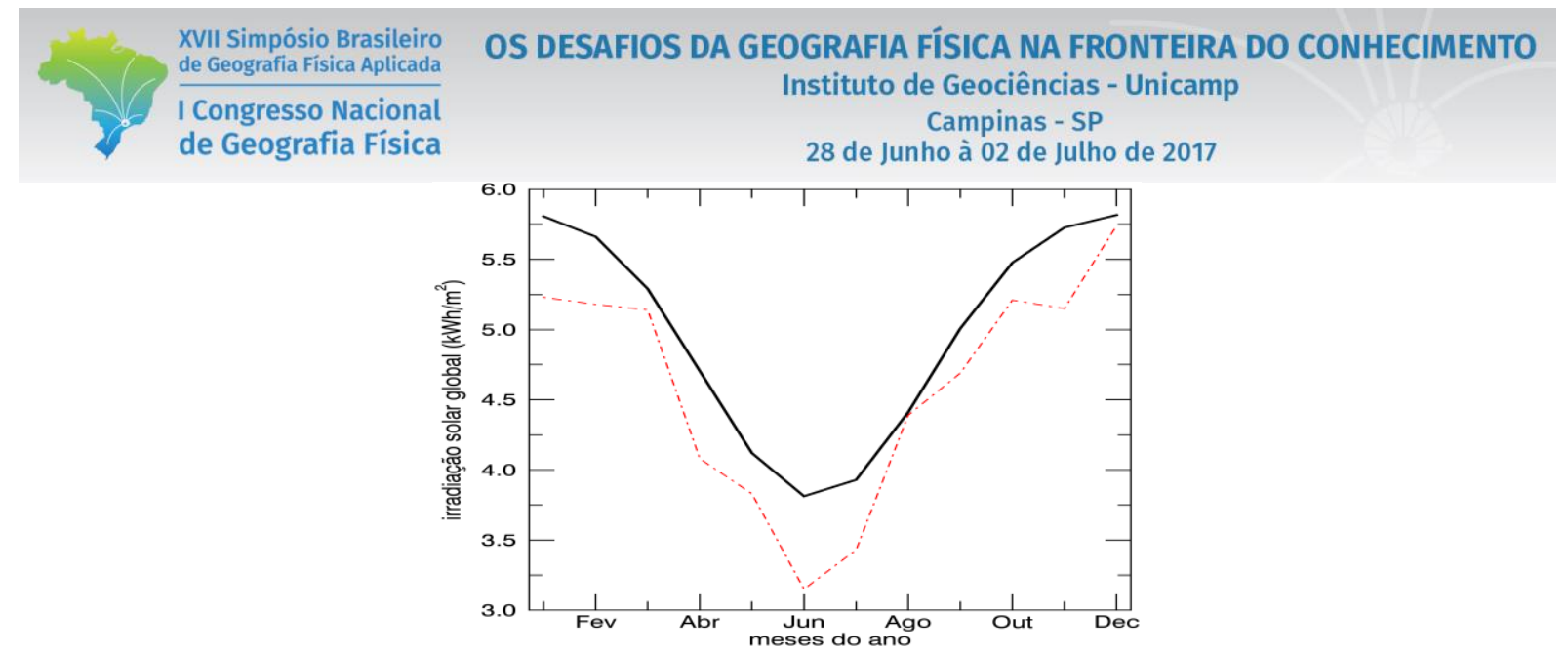

Figura 2 - Curva teórica e dos dados do PCD. Fonte: Dados de pesquisa

\subsection{Resultados esperados}

O resultado apresentado neste trabalho é bastante preliminar. Pretende-se, portanto, estender a análise aqui apresentada em diversas linhas, a saber: i) análise estatística temporal dos dados disponíveis no PCD de Ilhéus, para todos os anos possíveis, ii) ajuste adequado da curva teórica aos dados experimentais, considerando-se os desvios e incertezas pertinentes, iii) estimativa da distribuição espacial, com orientação e altimetria, da distribuição das coberturas residenciais no município de Ilhéus e estimativa do potencial de micro geração a partir da combinação destes dados com a evolução anual da irradiação solar global local. Desta forma, com o alinhamento de outras informações este projeto visa verificar a viabilidade para implantação de energia fotovoltaica no contexto da cidade de Ilhéus.

\section{Bibliografia}

AGENCIA NACIONAL DE ENERGIA ELÉTRICA (ANEEL). Resolução Normativa ANEEL no $\mathbf{4 8 2}$, de 17 de abril de 2012. Brasília, 2012. Disponível em: 〈http://www2.aneel.gov.br/cedoc/ren2012482.pdf〉. Acesso em 07 fev. 2017.

EMPRESA DE PESQUISA ENERGÉTICA (EPE). Disponível em <http://www.epe.gov.br>. Acesso em: 07 fev. 2017.

HERMANN, L. D.; CAMARA, I. P. Viabilidade de implantação de mini-usina de energia solar para o Campus Santo Ângelo/RS. Revista de Ciência e Inovação, v. 1, n. 1, 2016.

HONDA, D. Desempenho da usina fotovoltaica do campus Ceará-Mirim/IFRN. CONNEPI. Ceará-Mirim: Instituto Federal do Rio Grande do Norte, 2015.

INSTITUTO DO MEIO AMBIENTE E RECURSOS HÍDRICOS (INEMA). Monitoramento de PCDs. Salvador, 2011. Disponível em < http://www.inema.ba.gov.br/monitoramento/pcds/>. Acesso em 07 fev. 2017

INSTITUTO NACIONAL DE PESQUISAS ESPACIAIS (INPE). Sistema Integrado de Dados Ambientais. Disponível em <http://sinda.crn2.inpe.br/PCD/SITE/novo/site/index.php>. Acesso em: 07 fev. 2017.

ORDONES, R. Energia solar cresceu 70\% em dois anos. Rio de Janeiro: O Globo, 2017. Disponível em: <http://oglobo.globo.com/economia/energia-solar-cresceu-70-em-dois-anos-20715504>. Acesso em: 07 fev. 2017. 


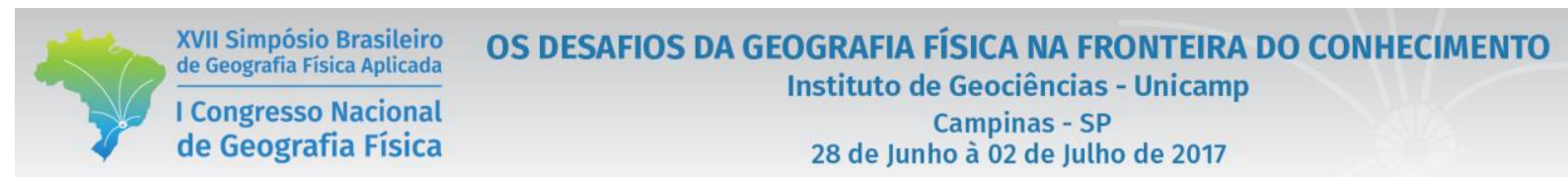

PEREIRA, E. B. et al. Atlas brasileiro de energia solar. Vol. 1. INPE, 2006.

SWERA, in Solar Wind and Energy Resource Assessment (dados disponíveis eletronicamente no endereço: http://en.openei.org/apps/SWERA/).

WANDERLEY, A.C.F.; CAMPOS, A.L.P.S. Perspectivas de inserção de energia solar fotovoltaica na geraçaõ de energia elétrica no Rio Grande do Norte. Holos, v.3 p. 3-14, 2013. 